\title{
Salivary cortisol determinations and self-rating scales in the assessment of stress in patients undergoing the extraction of wisdom teeth
}

\author{
C. M. Hill, ' and R. V. Walker ${ }^{2}$
}

\begin{abstract}
Objective To investigate salivary cortisol levels in patients undergoing third molar removal under local and general anaesthesia.
\end{abstract}

Design Random sampling of two independent groups.

Methods Samples of saliva were collected from patients prior to and after surgery for the removal of impacted wisdom teeth. The samples were analysed to assess cortisol levels. Patients were also asked to complete a Hospital Anxiety and Depression questionnaire on the day of surgery.

Results Patients receiving treatment under local anaesthesia showed lower levels of stress response than those having treatment under general anaesthesia on the actual day of surgery did (Fig. 1). No other significant differences were noted.

Conclusion Local anaesthesia is the preferred mode of pain control for patients who express no strong preference for type of anaesthetic.

Crom its introduction by Shannon and Prigmore, ${ }^{1}$ the non-inva-

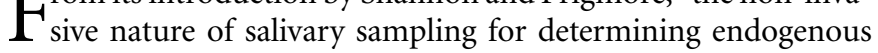
cortisol levels has been seen to be an advantageous technique in research on stress. ${ }^{2}$ The introduction of immunoassays by Walker et al. ${ }^{3}$ made such determinations more specific and cost effective and the greater sensitivity of the technique permitted smaller sample aliquots, thereby increasing the acceptability to patients. These advantages led to a widespread uptake of the technique, culminating in the publication of a review by Kirschbaum and Hellhammer ${ }^{4}$ and the publication of the proceedings of a workshop largely devoted to the role of salivary cortisol in research.

Interpretation of the results in stress research, however, has been complicated by interactions between the psychological and physiological components of stress. For example, in a marathon run, the massive rise in cortisol seen at about 15-18 miles is almost certainly a physiological response to impending hypoglycaemia, whereas the small rise seen just before the event (an anticipatory response) is psychogenic. When the 'threat' is purely psychological, such as in an important examination, results have been less clear. For instance, Cook et al. ${ }^{2}$ found an increased salivary cortisol immediately before the sitting of an examination, but not earlier on previous days (or even earlier the same day). There was, they proposed, an acutestress effect but not a response to chronic stress. This was also noted

\footnotetext{
${ }^{1 *}$ Consultant Oral and Maxillofacial Surgeon, ${ }^{2}$ Clinical Trials Coordinator, University of Wales College of Medicine, Dental School, Cardiff CF14 4XY ${ }^{*}$ Correspondence to: $C$. M. Hill

REFEREED PAPER

Received 13.10.00; Accepted 21.05.01

(c) British Dental Journal 2001; 191: 513-515
}

in volunteers taking part in a charity parachute jump where there was an extremely strong anticipatory response in raised salivary cortisol levels just before the scheduled jump. This suggested that the existence of a realistic chance of physical harm was likely to be a potent factor in the constitution of a reproducible stressor. Such considerations may suggest that anticipation of a surgical operation, particularly one where 'horror stories' abound, should be a reliable cause of stress.

The removal of wisdom teeth is currently a source of much research in the UK since nearly a quarter of a million teeth are removed every year at an estimated cost of over $\mathfrak{£} 70$ million. Within this group of patients lie fairly major differences since some may enter and leave having received treatment within an hour, whereas others may have to stay for up to four hours and sometimes longer depending upon the type of anaesthesia and any post-anaesthetic complications. Whilst some of this variation reflects a consideration of concurrent medical conditions, degree of difficulty and the patient's anxieties and preferences, much of it undoubtedly reflects our current ignorance; in particular whether the longer and more costly general anaesthesia protocol leads to a reduction in patient anxiety and stress. Although this would appear to be an obvious assertion, there appears to be no evidence in the literature that this is the case. To date, the use of salivary cortisol sampling as a measure of stress response does not appear to have been a technique used in oral and maxillofacial surgery, which is well known to involve procedures which patients find particularly stressful.

The purpose of this study was to use salivary cortisol determinations and self-rating scales to assess and compare stress and anxiety in patients undergoing extraction of wisdom teeth either by local or general anesthesia.

\section{Material and methods}

Fifty-four adult patients undergoing surgical removal of a unilateral impacted lower third molar ( \pm ipsi-lateral upper third molar) were recruited for inclusion in the study. The trial protocol was approved by the Local Research Ethics Committee and conformed to the Declaration of Helsinki. ${ }^{5}$ After discussion about the allocation of patients to either local anaesthesia or general anaesthesia, it was agreed that patient choice would be the only acceptable method of allocation despite the potential benefits of randomisation. It was agreed that such a method of allocation would be unlikely to introduce any bias into the actual results, however, since the immunoassays would be undertaken on a blinded basis by the biochemist. In addition, it was felt that all patients would probably select the mode of anaesthesia they felt most comfortable with.

An information sheet, explaining the purpose of the research, was given to patients asking them to collect samples of saliva each morning at approximately 0800 hours for seven days in total. This sam- 


\section{oral surgery}

Table I Salivary cortisol (mean \pm I S.D.); nmol/L and HAD scores

\begin{tabular}{llllll}
\hline A & $\mathrm{N}$ & pre-surgery & surgery & post-surgery & HAD scale \\
\hline LA & 27 & $24.6 \pm 14.9$ & $26.8 \pm 14.4$ & $20.7 \pm 11.1$ & $11.9 \pm 8.5$ \\
GA & 27 & $23.5 \pm 12.8$ & $36.2 \pm 15 . I^{*}$ & $23.6 \pm 14.2$ & $10.1 \pm 5.6$
\end{tabular}

*p $<0.00$ I: Students paired $t$-test

These results are also shown diagramatically in Fig. I.

pling regimen comprised of specimens collected on each of the three days before surgery, the morning of surgery and the following three days. Patients were also asked to complete a Hospital Anxiety and Depression scale (HAD) ${ }^{6}$ immediately prior to the operative procedure. Patients were divided into two groups; those receiving a local anaesthetic and those receiving a general anaesthetic during the surgical procedure. As mentioned, patients were allocated to the groups on the basis of their expressed preference whilst patients who were not given the option of free choice (for example, because of their medical history) were excluded.

The wisdom teeth were removed using a standard technique in both groups: access via a buccal muco-periosteal flap, bone removal with a bur, elevation, irrigation and placement of a single suture. When necessary, teeth were sectioned using a bur. The two groups were also analysed for degree of difficulty using MacGregor's 'WHARFE' method of scoring and length of surgical operating time. ${ }^{7}$

Patients were asked to collect saliva samples in small polypropylene vials and store them in a freezer pending their appointment. The saliva samples were collected in Sterilin ZS tubes as previously described $^{3}$ and the effect of freezing and thawing has been shown to cause no deterioration in the assaying technique. ${ }^{8}$

Subjects collecting mixed saliva were instructed to brush their teeth, rinse their mouth with water and wait 15 minutes before salivating directly into the collection tubes. Subjects found no difficulty in producing $3 \mathrm{ml}$ aliquots of mixed whole saliva in approximately five to ten minutes before the samples were frozen at $-4^{\circ} \mathrm{C}$ or less. Salivary cortisol concentrations were determined by direct radioimmunoassay, incorporating an I ${ }^{125}$ radio-ligand and an anti-serum raised in rabbits against a Cortisol-3-O carboxymethyl oxime, using a solid phase separation procedure as described by Cook et al. ${ }^{9}$

The data were analysed by standard statistical techniques comparing mean values using Student's $t$-tests.

\section{Results}

Comparison of the two groups using MacGregor's 'WHARFE' scores and surgical operating time showed no significant differences between the two groups.

Concentrations of cortisol in saliva samples collected pre-operatively, post-operatively and on the day of surgery in those subjects who underwent removal of wisdom teeth with either general anaesthetic (GA) or local anaesthetic (LA) are compared in Table 1.

In the GA group, no significant differences were observed when salivary cortisol concentrations in samples collected pre-surgery were compared with those observed in samples collected postsurgery. However, application of Students paired $t$-test indicated that salivary cortisol concentrations in samples collected on the day of surgery in subjects undergoing GA were higher $(p>0.001)$ than concentrations on either the pre- or post-operative days.

In the LA groups there were no statistical differences although the post-surgical levels showed a decreasing trend. No significant differences were observed in HAD scores recorded by subjects in the GA group when compared with those recorded by subjects in the LA group, although a trend to lower anxiety was noted in the GA group.

\section{Discussion}

Salivary cortisol sampling has been demonstrated to be an accurate and efficient means of measuring stress response. However, the ele-

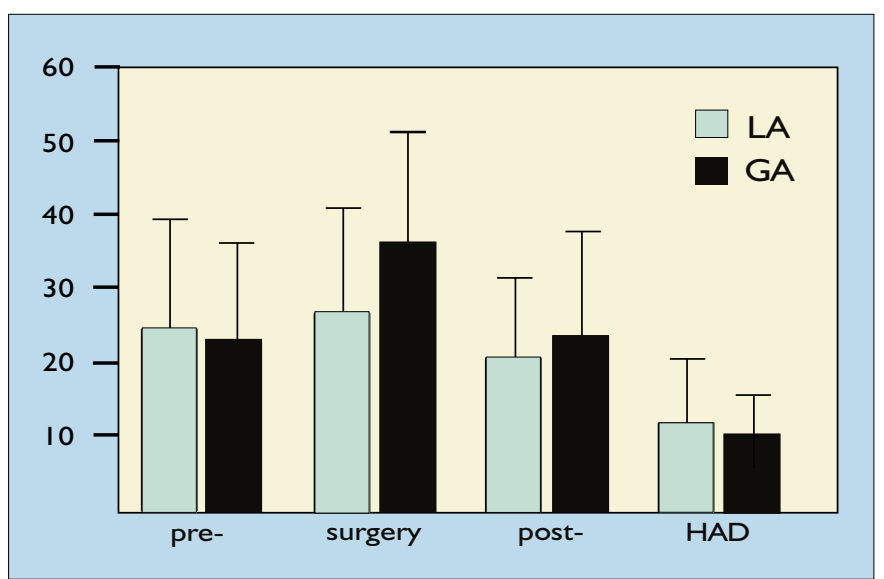

Fig. I Mean +I S.D. salivary cortisol concentrations (nmol/L) pre-, post- and on the day of surgery in subjects undergoing extraction of wisdom teeth under either general anaesthetic $(\mathbf{G A} ; \boldsymbol{n}=27)$ or local anaesthetic (LA; $n=27)$.

vation of salivary cortisol levels can be the result of both physiological and psychological stress as was previously mentioned. In the current research, we have not demonstrated any change in the stress responses of patients prior to surgery, but on the day of surgery there was a highly significant increase demonstrated in patients having treatment under LA. However, although this trend is notable in the group as a whole, the error bars indicate that the test would not be suitable for measuring the stress response of all individuals.

The overall results seem initially surprising but they perhaps reflect two factors. The first of these is that patients were only selected on the basis of their own preference for anaesthesia and it could be argued that the more anxious patients would tend to select general anaesthesia. The second possibility is that general anaesthesia (or the prospect of it) is indeed more stressful in that it is known by the general population to carry a higher risk of morbidity and mortality.

Paradoxically, however, these findings were not reflected in the HAD assessments, which showed a trend towards lower anxiety levels in the GA group.

There has been considerable debate recently questioning both the need for, and the means of the removal of, impacted wisdom teeth. ${ }^{10}$ Certainly there has been a move away from the extraction of symptomless, deeply impacted third molars thereby resulting in a relative increase in the number of teeth that are amenable to removal using LA. There is good evidence that treatment under LA is safer, cheaper and carries a lower morbidity. ${ }^{11}$ In addition, the National Institute of Clinical Excellence has recently published its guidelines on the removal of wisdom teeth. ${ }^{12}$ Despite all these changes in clinical practice, many patients (and indeed many surgeons) still prefer to complete their treatment under GA.

Regardless of any economic argument, this research suggests that patients who do not express a strong preference for GA should have their treatment completed under LA. In the future it would be interesting to expand this research to assess the possible benefits of sedation as a means of additional pain control. Clearly the minimisation of a stress response by whatever means is in the interest of both the patient and the surgeon.

\section{Conclusion}

The results of this study suggest that, where the patient has no preference for the mode of anaesthesia and in the absence of medical reasons, LA in an outpatient setting is the preferred mode of treatment both on economical grounds and in relation to the degree of stress experienced. The results also indicate the value of the stressfree, non-invasive nature of salivary sampling in the assessment of 
psychological and physiological stress. Stress response testing of individuals is unlikely to be a useful predictor of anaesthetic choice due to the variability in the assays.

1 Shannon I L, Prigmore J R. Parotid fluid as a medium for the determination of human adrenocortical status. Oral Surg 1960; 13: 878-882.

2 Cook N J, Read G F, Walker R F, Harris B, Riad-Fahmy D. Salivary cortisol and testosterone as markers of stress in normal subjects in abnormal situations. Gottingen: Hogrefe and Huber; 1992.

3 Walker R F, Riad-Fahmy D, Read G F. Adrenal status assessed by direct radioimmunoassay of cortisol in whole saliva or parotid fluid. Clin Chem 1978; 24: 1460-1463.

4 Kirschbaum C, Read G F, Hellhammer D H. Assessment of hormones and drugs in saliva in behavioral research. Gottingen: Hogrefe and Huber; 1992. 5 Declaration of Helsinki; 41st World Medical Assembly (1989)
6 Zigmond A S, Snaith R P. The Hospital Anxiety and Depression Scale. Acta Psychiatrica Scand 1983; 67: 361-70.

7 MacGregor A J. The Impacted Lower Wisdom Tooth. Oxford: Oxford University Press; 1985.

8 Campbell I T, Walker R F, Riad-Fahmy D, Wilson D W, Griffiths K. Circadian rhythms of testosterone and cortisol in saliva: effects of activity phase shifts and continuous daylight. Chronobiologma 1982; 9: 389-396.

9 Cook N, Harris B, Walker R F. Clinical utility of the dexamethasone supression test assessed by plasma and salivary cortisol determinations. Psychiatry Res 1986; 18 (2): 143-150.

10 Shepherd J P, Brickley M R Surgical removal of lower third molars. Br Med J 1994; 309: 620-621.

11 Brickley M R, Shepherd J P. An investigation of the rationality of lower third molar removal based on USA National Institutes of Health criteria. Br Dent J 1996; 180: 249-254.

12 Rawlins M. In pursuit of quality: The National Institute of Clinical Excellence. Lancet 1999; 353: 1079-1082. 